Available online on 15.09.2017 at http://jddtonline.info
Open Access to Pharmaceutical and Medical Research
noncommercial use, provided the original work is properly cited

\title{
ANALYSIS \& INTERPRETATION OF PREVALENCE OF PREDIABETES WITH SPECIAL REFERENCE TO COIMBATORE
}

\author{
Shanmugam and Rakesh Kumar Jat \\ Institute of Pharmacy, Shri Jagdishprasad Jhabarmal Tibrewala University Jhunjhunu Rajasthan, 313001, India
}

\section{ABSTRACT}

The Diabetes Mellitus patients glucose level alteration results were shown to few extent increased Glucose level with Diabetes Mellitus (18.7 \pm 0.0 ), Type-I Diabetes Mellitus (74.5 \pm 2.74$)$, T-II Diabetes Mellitus $(76.9 \pm 7.88)$ when make comparison with regular well fit volunteer $(123.7 \pm 1.953) \& 40-50$ years age groups of the patients were higher prevalence of Diabetes Mellitus (553 Numbers \& $36.9 \%$ ). Non Vegetarian are exaggerated more numbers \& percentage of Diabetes Mellitus (542 Numbers \& 36.1 $\%)$ when compare to Vegetarian (958 Numbers \& $63.9 \%$ ) \& also prominently affected DM with professional workers (540 Numbers \& $36 \%$ ). Comparatively $80-100 \mathrm{Kgs}$ body weight of Diabetes Mellitus patient's prevalence increased in Coimbatore zone. In Coimbatore zone T-I DM (470 Numbers \& $31.3 \%$ ) Patients prevalence were more T-II Diabetes Mellitus Patients (396 Numbers \& $26.4 \%$ ) \& non smokers were very less (544 Numbers \& $36.3 \%$ ). The result of inpatients indicated that theT-I Diabetes Mellitus prescriptions (228 Numbers \& 15.2\%) were more \& also out patients of T-II Diabetes Mellitus prescription (788 Numbers \& 52.5\%) were more (290 Numbers \& 19.4\%). The T-I DM proportional statement indicated Diabetes Mellitus associated Hyperlipidemic (38 Numbers \& $4.8 \%$ ), patient's prevalence was more \& also prescribed Poly therapy prescriptions (1155 Numbers \& $77 \%$ ). The results of prescribed tablet formulations were present in more numbers in the prescriptions when compared to other formulations.

Keywords: Diabetes mellitus, glucose, type I patients, type II patients.

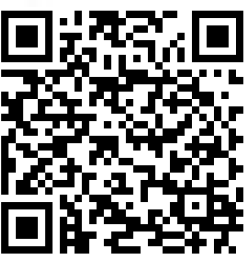

Article Info: Received 12 July, 2017; Review Completed 13 Sep, 2017; Accepted 13 Sep, 2017; Available online 15 Sep, 2017

Cite this article as:

Shanmugam, Jat RK, Analysis and interpretation of prevalence of prediabetes with special reference to coimbatore, Journal of Drug Delivery and Therapeutics. 2017; 7(5):94-101

DOI: http://dx.doi.org/10.22270/jddt.v7i5.1478

*Address for Correspondence

Shanmugam, Institute of Pharmacy, Shri JJT University Jhujhunu Raj-313001. Tel.: 9442216016, E-mail address: shans68@gmail.com

\section{INTRODUCTION}

Sort a couple of DM addresses positive piece of unending \& dynamic issue talking with heterogeneous issue brought on by totally extraordinary mixes of hypoglycemic specialist resistance \& reduced exocrine organ $\beta$-cell ability made by each innate \& obtained varieties from standard ${ }^{1-7}$. Polygenic illness is most basic metabolic issue that keeps on being not kidding even these days.

At this moment, kind a couple of DM is broke down while concealed metabolic varieties from standard involving hypoglycemic specialist resistance \& reduced $\beta$-cell ability cause stature of plasma aldohexose on top of $126 \mathrm{mg} / \mathrm{dl}(7 \mathrm{mmol} / \mathrm{L})$ in quick state or potentially on top of two hundred $\mathrm{mg} / \mathrm{dl}(11.1 \mathrm{mmol} / \mathrm{L})$ one $20 \mathrm{~min}$ when $75-\mathrm{g}$ aldohexose stack ${ }^{8}$. Yet described on commence of lifted plasma aldohexose levels, signally polygenic illness is envisioned by different associated varieties from standard. Every one of the a great deal of inside \& out, they slant affected public to outrageous genuine unlimited issues. Be that since it could, it's essential for medicative association to recognize "subclinical" polygenic illness to catch ailment technique. ${ }^{9}$

In any case, conviction that totally unique starting late investigated kind a couple of diabetic subjects beginning at present mastery appalling impacts of incite "late complexities of diabetes" at time of examination 10 demonstrates that assurance could are hold and, in like manner, that prediabetic condition is hurting to human achievement \& necessities created thought by executives \& general bounty. In this way, kind a couple of DM addresses essentially "tip of nippy mass" of long 
existing metabolic unsettling influences with hurting results for body structure, tissues, \& organs. Amid this technique, squeezing tries are required to abstain from making number of patients with this kind of "circumspectly executing" metabolic burden. ${ }^{11}$

Sixteen million individuals in United States with sort 2 diabetes mellitus \& additional 30-40 million with injured glucose versatility result in government disability costs outperforming 100 billion dollars dependably ${ }^{12}$.Treatment is overwhelmingly orchestrated at microvascular \& macrovascular challenges ${ }^{13}$. In sort 1 diabetes mellitus connection between glycemic control \& microvascular complexities has been settled in ${ }^{14}$. Connection between tight glycemic control \& micro vascular complexities has been settled in ${ }^{14}$. connection between tight glycemic control \& microvascular

clutter in sort 2 diabetes mellitus has all puts away being set up in starting late completed United Kingdom Prospective Diabetes Study ${ }^{15}, 16$. Bharat is immense country with masses isolating in racial admixtures moreover in social \& social affinities. It is likely that, significance of non insulin subordinate diabetes (sort 2 diabetes) could in like way be diverse in various races in light of trademark \& normal parts.

A conventional 1 million new cases are seen each year in public made $\geq 20$ years ${ }^{17}$. Two front line sicknesses, HIV-AIDS \& sort 2 diabetes mellitus, have pushed scorching calls for more achievable interventions. In 1980s, energetic spread of HIV, with its related true blue surprising infection \& high mortality, activated protester social affairs \& others to call for revived supporting of pharmaceuticals that displayed surety of credibility. ${ }^{18}$ Similarly, diabetes is viewed as general prosperity issue nowadays, \& it has been ordinary that number of diabetic patients are rising \& will rise from diagramed 135 million in 1995 to 300 million in $2025 .{ }^{19}$

Diabetes is more normal in African Americans, Latinos, Native Americans, \& Asian Americans/Pacific Islanders, besides made public. This understands they are in like path at increased hazard for making prediabetes. Inescapability of Type 2 diabetes is ending up noticeably out \& out \& moreover rapidly in Bharat as well. Asian Bharatns have ethnic deficiency to sort II diabetes. ${ }^{20}$ Type 2 diabetes constitutes around 85 to $95 \%$ of all diabetes in made countries, \& records for essentially higher rate in making countries ${ }^{21}$. There is gigantic declaration that, it is infection in various making \& beginning late industrialized nations ${ }^{22}$.

\section{MATERIALS \& METHODS}

Introduce cross advantageous examination will be depleted totally extraordinary ranges of Coimbatore. we will take once yank Diabetic Association (ADA) criteria for portrayal IFG and IGT. Obstructed quick aldohexose (IFG) was spoken to as quick plasma aldohexose estimation of $100-125 \mathrm{mg} / \mathrm{dl}(5.6-6.9 \mathrm{mmol} / \mathrm{L})$ while not past examination of polygenic sickness ${ }^{23}$. Incapacitated aldohexose Tolerance (IGT) was spoken to as plasma aldohexose array of $140-200 \mathrm{mg} / \mathrm{dl}$ ( 7.8 to $11.0 \mathrm{mmol} / \mathrm{L})$ 2 hours once oral alliance seventy five weight unit of aldohexose in subjects, whose plasma aldohexose obsession once long quick was underneath a hundred and forty $\mathrm{mg} / \mathrm{dl}$. (American polygenic ailment Association, 2007)

\subsubsection{Study reports engineering:}

Custom, ICF and CRF will be prepared. In technique Declaration of Helsinki religious principle was taken once. In wake of arranging, quality check will be performed. Required copies will be sorted out and submitted to ethics admonitory social capacity for its study. Educated consent structure will be got twist of as appeared by body might want.

\subsubsection{Case Record Form:}

An examination audit will be made feeling of a path to all association in subjects to assemble clear information.

\subsubsection{Demographic Details:}

Taking once statistic subtle components can gather from every single sharing subject.
a) Age
b) Sex
c) Height
d) Weight
e) BMI
f) Waist to Hip degree
g) Pulse Blood weight
h) Throb Blood weight

\subsubsection{Inclusion and Exclusion Criteria:}

\subsubsection{Inclusion Criteria:}

$\square \quad$ Men and young ladies more seasoned > twenty years

\section{Having case history of DM}

Subjects why should willing take eagerness for consider

\subsubsection{Exclusion Criteria:}

Patients UN office square allot diabetics and checking in wake of utilization timetable, movement and Oral side effect chiefs and hypoglycemic specialist beside. We will concentrate on relatives of diabetic patients since they rush to skill peril stratification and they agree to camp activities. to encourage around 1650 qualified subjects, approximately 1700 open (considering drop out level of around 15 August 1945, for any reasons) over $>20$ years later were screened from various degrees of Coimbatore. we tend to in like approach ensure that, we tend to inspire move to course from all cash associated classification, heading classification, field powers and working environment masters.

\subsubsection{Laboratory Investigations:}

Taking once examination work environment examinations will be performed for all open at central research facility. Qualified and planned power/phlebotomist can assemble blood tests.

a) Fasting glucose 
b) $\quad 2 \mathrm{~h}$ glucose

c) Total cholesterol

d) Serum Triglycerides

e) Serum cholesterin

f) Serum cholesterin

2.1.6 Ethics Committee Approval: consider records will be subjected to support by ethics Committee.

\subsubsection{Methodology took once in the midst of} Screening:

2.1.7.1 Activities finished before camps ( 2 weeks earlier):

$\square \quad$ Meeting with local Lions Club/Society/Community pack/School affiliation.

Meeting with local Family Physicians

Registration of subjects

\subsubsection{Activities finished in the midst of Camps:}

Validate day and age of subjects

Obtain consent from subjects

Collect blood for examinations (Fasting stage)

Administration of seventy five weight unit aldohexose confined in water

Meet ace (for physical examination) fervor for CRF

Collection of every statistic purpose behind

After a couple of hours blood gathering

At end, thought was made in occasion concerning, an approach to stop Diabetes?

\subsubsection{Anthropometric Measurement:}

Body weight will be measured (to closest a hundred gm) with subject halting on mensuration scale and weight correspondingly scattered on every leg. Subjects can instruct to wear littlest wear and no footwear, though their weight was being measured. Stature will be measured using no stretchable tape (to closest zero.1 $\mathrm{cm}$ ) with subject in erect position against surface and head engineered all together that prime of external sound-related way was in level with conventional edge of body circle. Body mass record will be found by disengaging weight (in kilograms) with sq. of stature (in meters). Midriff cutoff (to closest zero. $1 \mathrm{~cm}$ ) will be measured using tailor tape at reason mid-course between tip of bone pinnacle and last bone edge up back and at point some time recently.

After each subject are engineered five min, circulatory strain can measured twofold to closest a couple of mm $\mathrm{Hg}$ from left arm of half using ordinary weight gage. Ordinary of 2 estimations can use for all examinations. Throb circulatory strain (DBP) was recorded at fifth Korotkoff sound. experts finishes meeting audit, which can be coagulated sales worried past remedial history, case history of polygenic sickness, history of mending treatment, smoking inclinations, occupation and run the show. Smoking standing will be joined current smoker and non-smoker.

\subsubsection{Laboratory Analysis:}

Following 10-12 a hour of long sensible, each subject will be voided, and transient time allotment later quick biopsy will be amassed. seventy five weight unit anhydrous aldohexose disconnected in a couple of 50 metric limit unit of water will be given orally finished course of five min and second biopsy was drawn precisely $2 \mathrm{~h}$ later for aldohexose estimation. Blood for aldohexose assurance will be accumulated into tubes containing halide and EDTA. Amid think about one and only expert will be relegated to avoid social misconception. These blood tests will be unwisely centrifuged and prohibited any. Lipid profile tests will be poor around Erba EM 360 (Germany).

\subsubsection{Recruitment Status:}

In this erratically picked investigation, we tend to square gauge covetous to mix information for 1600-1700 subjects by discovering totally unique camps. Unobtrusive components of sped up screening camps square measure as shown by running with;

All subjects will be surveyed for Inclusion and Exclusion criteria as demonstrated by Protocol. No subjects will be picked coincidentally and by practicising that the larger part bewildering quality typical was kept up.

\subsubsection{Activities to be done once Camps:}

Discussion

Issue assurance and learning for various camps

Data space

Data affirmation and QC checks of data

Data change and examination

\subsubsection{Methodology took once in thick of data range:}

In thick of each camp, learning in CRFs can amass by implies that of sorted out partners. Time out there between quick aldohexose grouping and a couple of $h$ post aldohexose array will be used to gather finish and authentic statistic information. Each CRF will be similarly checked for its flawlessness and precision. Each partners can in like way offer wretched plan to avoid giving up vital open entryway fundamental and vital information. CRFs will be gathered and set away at secured and get to controlled spot. From that point forward, all CRFs will be assessed second time for any inconsistencies. at reason once check is finished, learning stage will be performed. Learning space will be performed exploitation Microsoft outperform 2003. Manual survey of CRFs will be performed to recognize irregularities, that can't be seen by electronic. 


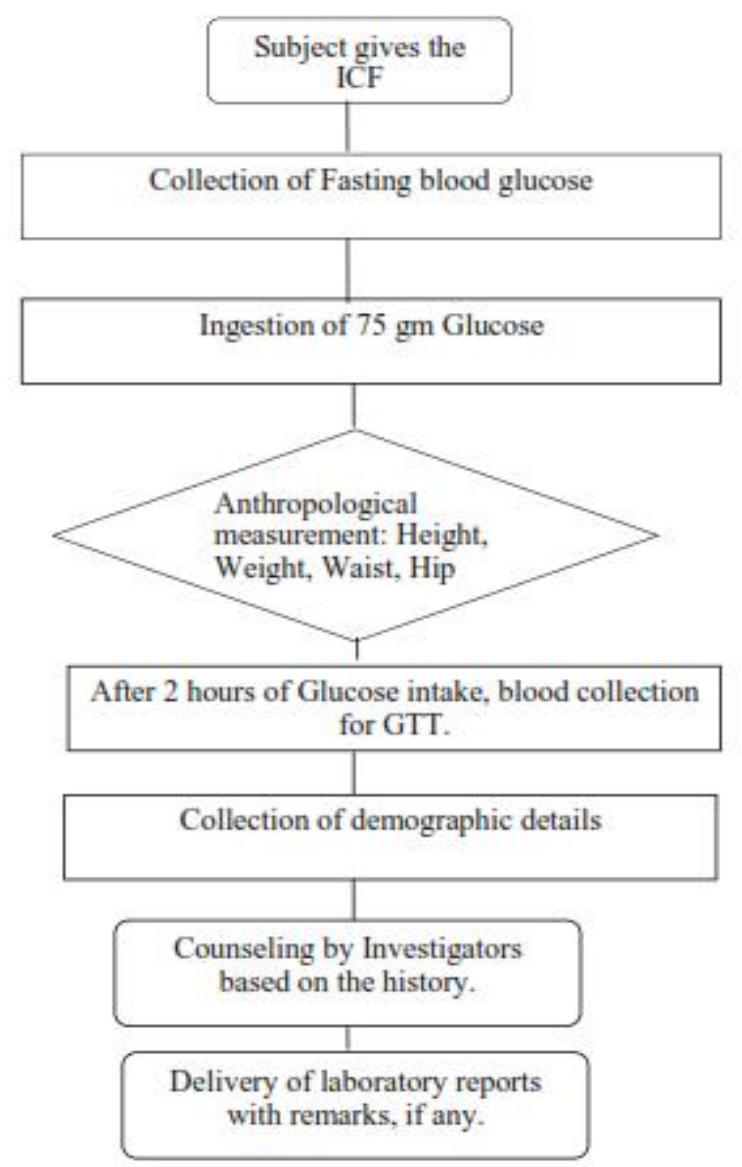

Figure 1: Schedule of events of trial.

\subsubsection{Methodology}

Took once in thick of data change and information examination: Quality survey (QR) will be performed to ensure learning uprightness and nature of data expectations. starting QR will be performed on got CRFs and lab reports. Wherever, we tend to felt learning square measure fantastical or irregularities, we tend to hurdle that information from examination.

\subsubsection{Statistical Analysis:}

Every single quantitative test will be viewed as fundamental at five-hitter level of essentialness. All learning will be reviewed by exploitation Chi sq. check exploitation SAS variety eight.2. Statistic, foundation and illustration information will be offered diagrammatically. Each and each persistent variable like age, height, weight and investigation work environment information was tended to by mean +SD (Standard Deviation). Each and each burdensome and snappy variable will be offered as checks and rates.

\subsubsection{Subjects \& setting}

The research work was approved by Institutional Human Ethics Committee (DCGI Reg. No. ECR / 668 / Inst / TN / 2014) \& also assigned approval number IHEC / 029 / Pharmacy / 08 / 2015. As per the standard guidelines subjects were selected \& studied different parameters demographic data such as age, sex, social history, family history, current treatment regimen, change of prescription drugs, current status of blood glucose level. Other parameters HDL, LDL, TCH, WBC, RBC, HB, SGOT, SGPT, PTP, sodium, potassium \& hematological variation were determined before \& after the administrating the drugs with DM patients. The above research work was carried out at Karpagam Medical College-Hospital at Coimbatore \& various multispecialty hospitals at Coimbatore.

\subsection{Study design}

Several literature survey based to selected Cohort study was used to estimate the various parameters of DM patients on various zone of Coimbatore which includes south, east, west \& north zones

\subsection{Sample Size}

Thousand five hundred DM patients were collected from Karpagam Medical College-Hospital \& various multispecialty hospitals, which include visited patients $\&$ out patients ${ }^{24}$.

\subsection{Study Criteria}

\subsubsection{Diverse criteria based to choose the patients}

\subsubsection{Glycemic Status}

Diabetes administration fixates on the patient, who accepts coordinate accountability though parts of his or her couldn't care less. This incorporates everyday administration of finger-stick glucose estimations, eating routine, work out, \& glucose-bringing down prescriptions (oral tablets as well as I infusions). Fruitful coordination of these factors is requesting \& entails incessant consideration.

Distinctiveness mediations appeared to enhance sugar manages, however difficult recognize the particular advantage of such intercessions \& unfocused impacts cram support, incorporate expanded patient consideration \& inspiration.

Nearby various confirmation facilitates individuals sugar manages would profit just commencing interest in irrefutable review is required managements DM patients.

We needed to appraise the impact of study interest sugar manages through review examination towards impact showings stopover facilitate goes before portion to managements irrefutable test.

Individuals conceivably qualified testing's rally review agent, for most review nurture. Over the span of stopover characters review is clarified, composed agree to cooperation is acquired \& pattern irrefutable research facility estimations subsist throughout. Counsel proposes parts of administration, for example, sugar observing, might likewise subsist advertised. Rebuff supplementary mediation has been existing and considered.

Qualification having been affirmed individuals has fetch flipsides moment event \& randomized innovative treatment. Deposits elsewhere's toward examine distinction in glycemic control, as deliberated by HbA1C, linking 2visits. Examination have been review individuals \& irrefutable groups partaking in these trials were unconscious that distinctions in sugar manages may subsist painstaking above the epoch amongst showings $\&$ randomization, in this way permitting us to look at the impact of enrollment to a irrefutable 
experiment upon sugar manages commencing adjustment on treatment.

\subsubsection{Look into Design \& Method for clinical study}

Corporation's outlines \& does randomized forbidden audition toward innovative treatments \& additionally innovative regimes for sort -I \& sort- II DM \& accordingly substantial catalogs for examination.

Qualified tryouts subsist main randomization solitary stopover reason for review had been clarified, educated assent have gotten \& taken taxing; pharmacy treatment has unaltered amongst showings \& randomization; \&) HbA1C deliberated similar research facility on every 1. event. Of the irrefutable tryouts directed through support worldwide advancement lineup for lipro, 3 tryouts (supported method) Individuals amid sort-I DM \& 3tryouts in individuals amid sort-II DM led somewhere around nineteen ninety nine \& two thousand one, congregate on consideration criterion.

Individuals 3 tryouts sort-IDM originated from six European nations, \& the 3tryouts sort- II DM were led in the Choice criterion on sort-I DM \& sort- II DM contemplate bunches contrasted sort-I DM studies generally acceptable sugar manages, while in sort-II DM studies were distinguished on the premise towards underprivileged sugar manages. Individuals enrolled tryouts sort -I DM have matured one eight-seven five yrs, have irrefutable finding towards sort-II DM \& every subset 4 every day infusions human Insulin. Individuals amid unfortunate sugar manage prohibited \& characterized $\mathrm{HbA} 1 \mathrm{C}$ greater than one point five times furthest reaches no Diabetes Mellitus go. Members on 3trials have been accordingly randomized towards unfasten otherwise canopied examinations towards Insulin lspro amid routine creature Insulin.

Epoch criterions for tryouts on sort-II DM shifted from forty to eight five yrs (learning's), two five to eight five yrs (consider) \& eight five to seven five yrs (examine). Every subsist captivating verbal sugar-bringing down treatment intended hyperglycemia \& have been imperfect sugar manages. Consider included individuals amid sulfonylurea's disappointment characterized on HbA1C get eight point five percentage; contemplate included patients with $\mathrm{HbA1C}$ get eight point zero percentage in spite of managements amid no less than solitary verbal sugar-bringing down operator; \& lessens included individuals amid $\mathrm{HbA} 1 \mathrm{C} \geq 8.0 \%$, in spite of treatment with no less than two oral glucose-bringing down specialists. Ensuing randomization have mix verbal treatment, every day in addition to glyburide, or thrice-day by day lspro in addition to glyburide (contemplate); towards twofold otherwise trice day by day Insulin (think about); \& towards once-day by day in addition to or a thrice-day by day blend in addition to metformin (examine F). Of six trials incorporated into this investigation, concentrates \& distributed during occupied, \& college work \& distributed dynamic frame .Free oral pharmaceutical, yet not glucose-observing gear, have been accessible towards individuals 3reviews in sort-II DM amid keep running epoch.
The exchange off between sum up capacity \& inner legitimacy for clinical reviews Clinical reviews are central for deciphering leaps forward in fundamental human wellbeing.

At the point when selecting study members, analysts must consider logical, moral, administrative, \& wellbeing necessities \& make an interpretation of these into unambiguous qualification criteria.

Be that as it may, excessively prohibitive member choice has bargained consider sum up capacity, seriously weakened the money saving advantage.

\section{RESULTS \& DISCUSSION}

\subsection{PREDIABETES:}

Pre-diabetes (incorporate IFG and IGT) is expressed that happens once person's glucose levels are past customary yet not enough high for assurance of polygenic issue.

terms IFG and IGT guide direct metabolic stage between standard aldohexose and polygenic issue. thought of IFG was shown by Charles et al. to direct at restraint plasma aldohexose levels $>$ half dozen. 1 and $<7.8 \mathrm{mmol} / \mathrm{L}^{25}$. Diversion aldohexose adaptability is all extra best in school measure of modification in aldohexose absorption structure than blocked restraint aldohexose ${ }^{26}$. These forbearance plasma aldohexose limits qualification with level on top of that genuine stage hypoglycemic specialist surge is lost as aftereffects of endogenous relationship of aldohexose.

Around eleven p.c of open with pre-diabetes in polygenic turmoil impediment Program ordinary or administration bunch made sort a couple of polygenic issue efficiently in within standard 3 years of post liminary. Particular examinations outline, that various open with pre-diabetes fabricate sort a couple of polygenic issue in ten years. starting late, proficient Committee on recognizable proof and Classification of diabetes (1999) anticipated diminishing in each higher and lower restraint aldohexose esteems for examination of IFG to relate at new finding criteria for polygenic confusion.

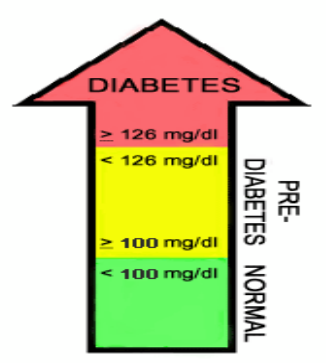

FPG

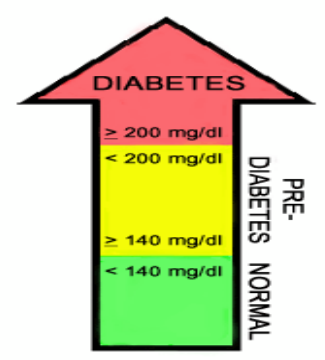

OGTT

\section{Figure 2: Blood glucose ranges in IFG \& IGT patients}

Regardless, late reports have shown gather activity of recognition between 2-h post aldohexose depiction of IGT and forbearance aldohexose class of IFG among subjects of grouped ethnic open groups. Among North American country adults, 40-74 years later, 15.6 p.c (14.9 million) have IGT, and 9.7 p.c (9.6 million) have 
IFG. signally, examination on 7-year follow-up Funagata polygenic confusion Study incontestable that IGT is risk half for vessel poor shape, yet IFG isn't ${ }^{27}$. In addition, parts basically based examinations have demonstrated higher affectability of IGT over IFG for envisioning headway to sort a couple of polygenic issue.

People with pre-diabetes are at higher danger of vessel infirmities. Individuals with pre-diabetes have one.5overlay danger of vessel sullying risen up out of individuals with normal glucose. Individuals with polygenic confusion have a couple of to four overlay created risk of vessel diseases. Individuals WHO are overweight and age forty five or extra set they up, must be constrained to be checked for pre-diabetes in the midst of their next routine medicinal visit. In occasion that their weight is standard and they're over age forty five, they must be constrained to request their expert in center from routine working environment visit, if testing is right.

For adults more youthful than forty five and overweight, your star could direct testing on off likelihood that you essentially have regardless of various threat fragments for polygenic turmoil or pre-diabetes. These join hypertension, low cholesterol and high triglycerides, case history of polygenic issue, history of physiological state polygenic confusion or exchange youthful measure very four kilogram ( nine pounds) or having spot with ethnic or minority pack at high hazard for polygenic turmoil.

To keep up organic liquid aldohexose underneath seven. $8 \mathrm{mmol} / \mathrm{L}$, subjects with IFG demonstrate basic hyperinsulinemia post aldohexose stack, however IGT subjects show low hypoglycemic operator radiation not prepared to remain up natural liquid aldohexose inside typical recorded achieve esteems, disclosures that propose particular circumstances of hindered hypoglycemic specialist release.

On same technique, our exposures demonstrate that IGT is extra best in educational time of congruousness in aldohexose maintenance framework than IFG. One reason of this introduction, assemble activity of concordance among IFG and IGT criteria that has been addressed.

IFG and IGT are metabolically clear diffuses with influenced unfurl, among all inclusive community WHO had IFG and additionally IGT, sixteen p.c had each IFG and IGT, twenty three p.c had IFG alone and sixty p.c had IGT alone.

IGT is foremost condition that by and enormous makes danger for coming back to realization adjustment of sort a couple of polygenic issue. Open with IGT demonstrate varieties from typical in each hypoglycemic specialist improvement and early hypoglycemic operator release like those found in patients with sort a couple of polygenic issue. These varieties from typical not just go before polygenic confusion, they accept it as well. Additionally, change from IGT to polygenic turmoil is envisioned by uncommon diminishing in early hypoglycemic operator release. it's these days clear that early hypoglycemic specialist unharnessed recognize basic half in smart and persuading cowl regarding endogenous aldohexose creation taking once supper. Loss of early hypoglycemic specialist surge at first prompts postprandial side effect that, as unwellness propels, decreases to clinical side effect.

Considers have demonstrated that individuals with prediabetes will stay away from or put off advance of sort a couple of polygenic issue by up to fifty eight p.c through changes to their technique forever that harden clear weight decreasing and ordinary improvement. ace board recommends, that individuals with pre-diabetes diminish their weight by 5-10 p.c and give in some sensibly humble physical headway for half-hour directed. For couple individuals with pre-diabetes, interceding early will truly flip back clock and come raised glucose levels to basic degree.

\subsubsection{Pre-diabetes harbinger of future polygenic issue}

Disabled aldohexose quality (IGT) and impaired fasting aldohexose (IFG) in lightweight of current conditions alluded to as pre-diabetic states, have high threat of headway to diabetes. couple appearance at have shown that these pre-diabetic states are to boot high risk stages for cardiovascular disease ${ }^{26,27}$. amid this strategy information on IGT and IFG are other than urgently required as they're indicators of future polygenic issue simplification and weight on the nation. NUDS comes about show that prevalence of IGT was past that of sort a couple of polygenic issue in four out of six urban social occasion's studied $^{13}$. sweeping statement of IGT was sixteen. 8 for each penny in city, 14.9 for every penny in Bangalore (once in past Bangalore), 29.8 for every penny in Hyderabad, ten for every penny in Kolkata, 10.8 for each penny in Mumbai and eight. 6 for every penny in New Delhi. ADEPS done in Kerala incontestable that eleven. 2 for each penny of the subjects had either IFG or IGT17. PODIS reported that constrain of IGT was unexceptionally fundamental level high in each customary and urban populations ${ }^{22}$. late investigation has concurring lessened all inclusive statement of IGT in urban parcels risen up out of before examines depleted same city ${ }^{19}(16.8 \%$ in 2000 to ten.2\% in 2004)(Fig. 5). this may recommend that polygenic issue pandemic in urban Asian nation may well be backing off or it would also suggest that there may well be lively change from normal state through IGT to polygenic confusion, that could imply quick development in polygenic turmoil torment or worsening diabetogenic environment.

\subsubsection{Symptoms of hypoglycemic operator Resistance and Pre-diabetes}

Insulin resistance and pre-diabetes for some half haven't any reactions. In occasion that you just have incomprehensible sensibly hypoglycemic operator resistance, you'll get black out patches of skin, all around on back of your neck. As not particularly dependable religious principle individuals get exhausting ring around their neck. diverse possible goals for these powerless patches wire elbows, knees, knuckles, and armpits. This condition is named skin issue. Fernando R. 


\subsubsection{Pathogenesis of Prediabetes}

Progression from ordinary aldohexose quality to sort a couple of polygenic issue is depicted by twofold surrenders that are a piece of hypoglycemic specialist resistance and hypoglycemic operator secretary contorting made by beta-cell brokenness.

Progression to Type 2 Diabetes

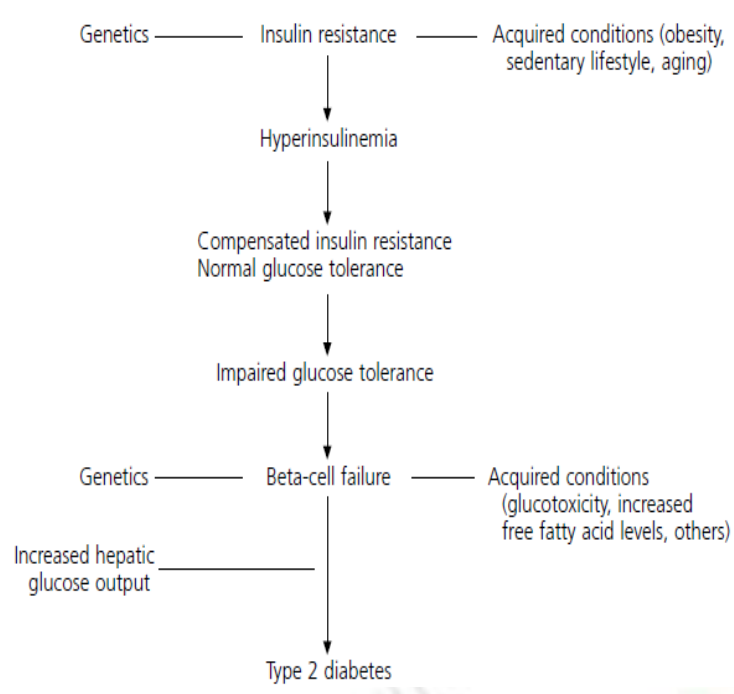

Figure 3: Etiologic sequence for development of type 2 diabetes.

Insulin resistance is envisioned by lessened tissue affectability to hypoglycemic operator and checked neutralizing hyperinsulinemia. At in any case, plasma aldohexose levels are kept up in keep running of plant reach. In patients, WHO can eventually fabricate polygenic confusion, there's alteration in beta-cell secretary constrain.

Insulin resistance goes before onset of sort a couple of diabetes by imperative augmented measure of your time. In within now prediabetic condition of repaid hyperinsulinemia exists depict by barred aldohexose adaptability and identified with dyslipidemias and diverse metabolic assortments from typical of hypoglycemic operator resistance state.

Closeness of long history of atherogenic, prediabetic state stresses significance of entire arrangement system for basic avoidance of sort a couple of polygenic issue.

Regardless aldohexose choice from typical that is perceived is ascend in postprandial aldohexose levels because of decreased first-compose hypoglycemic operator unharnessed. With time, extra reduction in cell restricts prompts stature of restraint aldohexose levels. Finally, polygenic confusion happens, with extra hypoglycemic operator secretary hardship.

\section{Focus on Prevention:}

IGT is first just unmistakable stride in path physiology of sort a couple of diabetes. it's identified with high hazard for sort a couple of diabetes and following tube foulness and mortality. it's these days dim, regardless of whether treating IGT can reduce rate of full scale tube shortcomings, as concentrates watching out for the present issue have however to be driven. Thus, essential motivation to recognize and treat IGT is to stay off from or yield onset of sort a couple of diabetes.

Late examinations of patients with IGT have shown activity for modus Vivendi mediations in consent or retribution progress of polygenic issue.

There is solid confirmation that made attempt out of utilization program and activity will reduce danger of improvement to sort a couple of polygenic issue in patients with IGT. Patients with IFG and IGT should be mentored on good conditions concerning straightforward weight diminishment, unprecedented dietary slants and unfaltering physical improvement.

IGT is portray by improvement in postprandial aldohexose levels, that is viewed as soonest metabolic choice from ordinary in sort a couple of diabetes. it's one among mastermind of danger fragments for CVD (hypertension, high lipid levels, low high-thickness lipoprotein-cholesterol and focal power), called metabolic issue. various parts making up this issue are by and by associated. destroyed super molecule profile will help hypoglycemic specialist resistance, as IGT could make do with horrific half on various vessel risk components.

Macronutrients (MNT) are vital section of polygenic issue slacking, association, and association preparing. Notwithstanding its half in avoiding and prevailing polygenic issue, ADA sees position of sustenance as significant part of general tough modus Vivendi.

Clinical trials/result examinations of MNT have concurring reductions in $\mathrm{A} 1 \mathrm{C}$ at 3-6 months going from zero. $25 \%$ to $2.9 \%$ with higher decreases found in sort a couple of polygenic issue of shorter term.

\section{SUMMARY \& CONCLUSION}

This was introductory illustrative examination provoked review consensus of Prediabetes in Coimbatore. In this way, it's driven most preeminent criticality, as until the point when these days we keep an eye on range unit depending upon various disseminations from various tad bit of India and abroad.

From this investigation we tend to see that, general greatness of IFG in Coimbatore is around two.72\% and IGT is around four.67\%. Additionally, basically watched that, prediabetes typicality is expanding directed in urban and ordinary zone as eventual outcome of progression in mold, sustenance slant, working society, wealth.

In the event that we have a tendency to extrapolate with add up to loads of state, Coimbatore can have be around one.31 million open of IFG and two.30 million open with IGT territory unit available comfortable time. to boot, on the off chance that, we have a tendency to recognize same quantum of ascend, than in next ten years these numbers may wind up being very twofold i.e. around four million of IFG and five million of IGT alone. close that, on the off chance that we have a tendency to recognize alteration rate from IFG and IGT to polygenic turmoil, than every 2-3 years around eighty,000-140,000 new polygenic issue range unit 
consolidated to current open. this is frequently obstacle in economy of state and country, as enthusiastic parcels is changed over into polygenic turmoil.

Lipid irregularity is in like way announced amid this parts obtainable aldohexose determination from typical. This obviously delineates, amid this open obtainable aldohexose, we tend to need to target lipoid choice from typical revisions. Clinicians normally concentrate basically on aldohexose, withal we tend to need to target lipoid conjointly, to remain up essential division from progress of polygenic issue dyslipidemia could likewise

\section{REFERENCES}

1. Gerick J., hereditary premise of sort a couple of polygenic issue mellitus: debilitated interior discharge emission versus hindered inner discharge affectability, Endocr. Rev. 1998; 19:491-503.

2. DeFrantz R., (1992), pathologic procedure of sort a couple of (non-insulin subordinate) polygenic confusion mellitus: adjusted outline, Diabetologia, 35, 389-97.

3. Yki-Järvinen H., (1994), pathologic procedure of ketoacidosis-safe diabetes mellitus, Lancet, 343, 91-5.

4. Ferrannini E., (1998), inner discharge resistance versus inside emission insufficiency in noninsulin-subordinate polygenic issue mellitus: issues and prospects, Endocr. Rev., 19, 47790.

5. Kahn C., Bunting Lecture: inward emission activity, diabetogenes, and explanation behind sort II polygenic confusion, Diabetes, 4, 1066-84.

6. Olefsky J., interior emission resistance and pathologic procedure of noninsulin-subordinate polygenic issue mellitus: cell and atomic systems, Adv. Exp. Med. Bio., 334, 129-50.

7. Haring H.,(1999), pathologic procedure of sort II diabetes: are there regular foundations for interior emission resistance and discharge failure.,Exp. Clin. Endocrinol. Diabetes., 107(2), S17-23.

8. Harris M. and Cowie C., (1998), Prevalence of polygenic issue, weakened forbearance aldohexose, and disabled aldohexose resistance in U.S. grown-ups, polygenic confusion Care. 21, 518-24.

9. Greogory A. and Brown., (2007), Progression from new no inheritable disabled restraint aldohexose to sort a couple of polygenic issue, polygenic turmoil Care, 30, 228-33.

10. Ramachandran A. and Vijay V., (2002), Diabetic investigation. and Clinical. Practice, $58,55-60$.

11. Hayden M., (2002), , Journal Of Pharmacy, 3 , $126-38$.

12. Kahan S. and Priogeon R., (1993), Quantification of connection between insulin-affectability and beta-cell work in human subjects, Diabetes, 42, 1663-72.

13. Kahne S., (2000), significance of beta-cell in pathologic procedure of sort a couple of DM, Am. J. Med., 108(6), 2S$8 \mathrm{~S}$.

14. Harris M. and Couric C., (1995), polygenic turmoil in America, 95(2), 1468. be arrange are frequently named pre dyslipidic/metabolic perplexity.

We moreover must be constrained to move parts, World Health Organization region unit drawing closer at forty years in light-weight of undeniable truth that amid this age we tend to establish most convincing energy of IGT. Certainly, even clinically in like way when this age, downsized scale vascular changes begins and could likewise be bolster with obnoxiousness. As un favorableness increases to the present age pack it's gigantic effect on pharmaco economical stack on individual and at country level.

15. Mohan., (2007), solution of sort a couple of Diabates, Bharat. J. Med. Res., 12(5), 217-30.

16. Raman K. and Joseph A., (1999), High commonness of sort a couple of polygenic issue inurban settlement in Kerala Asian nation, Ethn. Wellbeing, 4 , 231-9;.

17. Shah S. and Saikia M., (1999), High commonness of sort a couple of polygenic issue in urban populace in north jap Asian nation, Int. J. Diabetes. Dev. Nations, 19, 144-7.

18. Zargar A. and Bashir M., (2000), Prevalence of sort a couple of polygenic issue mellitus and hindered aldohexose resistance in geographic locale vale of Bharat landmass, Diabetes. Res. Clin., 47 , 135-46.

19. Ramachandran A. and Snehalatha C., (2001), 44, 1094-101.

20. Almdal T. and Scharling H., (2008), Higher predominance of hazard elements for sort a couple of DM and resulting higher frequency in men, Eur. J. Assistant. Med., 19(1), 40-5.

21. Zafar I. and Abdul B., (2010), Dietary Patterns identified with Risk for Metabolic Syndrome in Urban Community of urban focus plot by Cluster Analysis, Pakistan J. of Nut., 9 (1), 93-9.

22. Abbas A., 2008), polygenic turmoil screening in Basrah, Iraq: populace essentially based cross-sectional examination, polygenic confusion Res. Clin. Pract,. 79., 147-50.

23. Ajagaonkar A., (1966), polygenic confusion in Asian nation, Man. Med. J., 14-6.

24. Ajagaonkar S., (1972), DM as found in antiquated Ayurvedic solution in inside discharge digestion, 1-19.

25. Amina K. (2011), Prevalence and hazard elements of polygenic issue and impeded forbearance aldohexose in Nauru, BMC Public Health, 11, 719.

26. Anjana R., (2011), Prevalence of polygenic issue and prediabetes (hindered restraint aldohexose and additionally debilitated aldohexose resistance) in urban and provincial Bharat: clinical test aftereffects of Bharat Council of Medical Research-Bharat polygenic turmoil (ICMR-BHARATB) think about., Diabetologia, 54(12), 3022-7.

27. Shivananda N., (2011), Association of low funniness creatinine, unusual lipide profile, sexual orientation, age and quality with sort a couple of DM in Trinidad and island, polygenic confusion Res. Clin. Pract., 91, 342-7. 